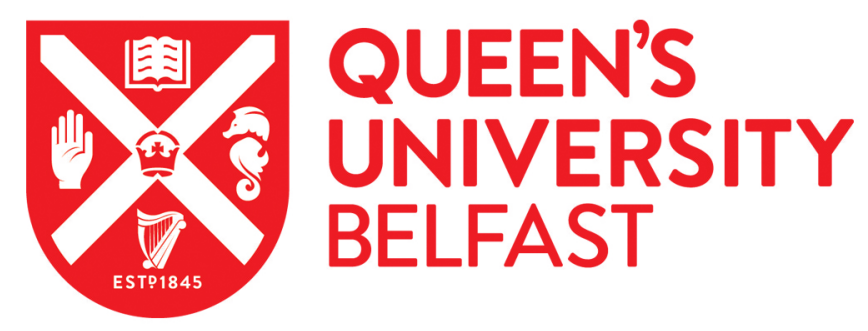

\title{
The radiobiology of laser-driven particle beams: focus on sub-lethal responses of normal human cells
}

Manti, L., Perozziello, F. M., Borghesi, M., Candiano, G., Chaudhary, P., Cirrone, G. A. P., Doria, D., Gwynne, D., Leanza, R., Prise, K. M., Romagnani, L., Romano, F., Scuderi, V., \& Tramontana, A. (2017). The radiobiology of laser-driven particle beams: focus on sub-lethal responses of normal human cells. Journal of Instrumentation, 12, [C03084]. https://doi.org/10.1088/1748-0221/12/03/C03084

Published in:

Journal of Instrumentation

Document Version:

Peer reviewed version

Queen's University Belfast - Research Portal:

Link to publication record in Queen's University Belfast Research Portal

Publisher rights

(c) 2017 IOP Publishing Ltd. \& Sissa Medialab srl. This work is made available online in accordance with the publisher's policies. Please refer to any applicable terms of use of the publisher.

\section{General rights}

Copyright for the publications made accessible via the Queen's University Belfast Research Portal is retained by the author(s) and / or other copyright owners and it is a condition of accessing these publications that users recognise and abide by the legal requirements associated with these rights.

Take down policy

The Research Portal is Queen's institutional repository that provides access to Queen's research output. Every effort has been made to ensure that content in the Research Portal does not infringe any person's rights, or applicable UK laws. If you discover content in the Research Portal that you believe breaches copyright or violates any law, please contact openaccess@qub.ac.uk. 


\title{
The radiobiology of laser-driven particle beams: focus on sub-lethal responses of normal human cells
}

\author{
L. Manti, ${ }^{\mathrm{a}, 1}$ F. M. Perozziello, ${ }^{\mathrm{a}, 1}$ M. Borghesi, ${ }^{\mathrm{b}}$ G. Candiano, ${ }^{\mathrm{c}}$ P. Chaudhary, ${ }^{\mathrm{d}}$ G.A.P. \\ +- Cirrone, ${ }^{\mathrm{c}}$ D. Doria, ${ }^{\mathrm{b}}$ D. Gwynne, ${ }^{\mathrm{d}}$ R. Leanza, ${ }^{\mathrm{c}}$ K. M. Prise, ${ }^{\mathrm{d}}$ L. Romagnani, ${ }^{\mathrm{e}}$ and \\ A.Tramontana ${ }^{\mathrm{c}}$
}

\author{
${ }^{a}$ Physics Department, University of Naples Federico II, \& INFN, Naples Section \\ Complesso Universitario di Monte S. Angelo, Via Cintia, 80120,, Naples, Italy \\ ${ }^{b}$ Centre for Plasma Physics, School of Mathematics and Physics, Queen's University Belfast \\ University Road, BT7 1NN, Belfast, United Kingdom \\ ${ }^{c}$ LNS-INFN, ViaS. Sofia 62, 95125 Catania, Italy \\ ${ }^{d}$ Centre for Cancer Research and Cell Biology, Queen's University Belfast, \\ University Road, BT7 1NN, Belfast, United Kingdom \\ ${ }^{e}$ LULI, Ecole Polytechnique, CNRS, CEA, UPMC, 91128 Palaiseau, France.
}

E-mail: manti@na.infn.it,francescaMP@hotmail.it

\begin{abstract}
Accelerated proton beams have become increasingly common for treating cancer. The need for cost and size reduction of particle accelerating machines has led to the pioneering investigation of optical ion acceleration techniques based on laser-plasma interactions as a possible alternative. Laser-matter interaction can produce extremely pulsed particle bursts of ultra-high dose rates $\left(\geq 10^{9} \mathrm{~Gy} / \mathrm{s}\right)$, largely exceeding those currently used in conventional proton therapy. Since biological effects of ionizing radiation are strongly affected by the spatiotemporal distribution of DNA-damaging events, the unprecedented physical features of such beams may modify cellular and tissue radiosensitivity to unexplored extents. Hence, clinical application of laser-generated particles needs assessment of their radiobiological effectiveness. To date, the majority of studies have either used rodent cell lines or have focussed on cancer cell killing being local tumour control the main objective of any radiotherapy strategy. However, very little data exist on sub-lethal cellular effects of relevance to normal tissue integrity such as premature cellular senescence. Here, we briefly discuss ultra-high dose rate radiobiology and present preliminary data obtained in normal human cells following irradiation by laseraccelerated protons at the LULI PICO2000 facility at Laser Lab Europe, France.
\end{abstract}

KEYWORDS: Laser-driven proton beams; Radiobiological effectiveness; Sub-lethal effects and premature cellular senescence.

\footnotetext{
${ }^{1}$ Corresponding author
} 


\section{Contents}

1. The rationale for laser-driven cancer hadrontherapy

2. The radiobiology of ultra-fast high-energy particles

Error! Bookmark not defined.

3. Sub-lethal cellular damage and particle therapy

Error! Bookmark not defined.

4. Radiobiological studies with laser-accelerated protons at the $\mathrm{PICO}_{2000}$ facility

4.1 Experimental setup

4.2 Results

4.3 Conclusions

\section{The rationale for laser-driven cancer hadrontherapy}

Hadrontherapy, the clinical exploitation of accelerated charged particles for cancer treatment, has become an increasingly common therapeutic modality [1], because of several advantages it offers compared to conventional radiotherapy with megavoltage photon or electron beams. The use of charged particles significantly reduces the dose absorbed by normal tissues due to their inverse dose-depth deposition profile as described by the Bragg curve. Their superior ballistic properties are thus the physical pillar justifying hadrontherapy as the eligible treatment for deepseated tumours and/or close to organs at risk [2]. Heavier charged particles such as carbon ions exhibit a higher radiobiological effectiveness than protons or photons, due to their denser ionization event pattern through matter, whereby highly clustered DNA lesion sites arise. Such damage complexity is believed to confer high-LET radiations their radiobiological advantage at cell killing [3], hence making carbon-ion based radiotherapy best suited to treat intrinsically radioresistant cancers [4]. However, a wider adoption of hadrontherapy is hampered by the high installation and running costs of the accelerating facilities [5]. This has fuelled efforts in developing novel approaches to obtain a compact, flexible and cost-effective solution to accelerate particles for medical applications. One viable solution may be represented by laserbased optical ion acceleration techniques [6]. Clinically amenable laser-driven beams require specific constraints to be met, such as beam reproducibility, homogeneity and stability. More importantly, the radiobiological effectiveness of these beams needs must be assessed. For they exhibit unprecedented spatio-temporal physical features [7]. The physical pattern of the energy deposition events at nanometric scale and their subsequent very early stage $\left(10^{-12}-10^{-6} \mathrm{~s}\right)$ radiochemical evolution determines the biological outcome, shaping the role of biochemical repair processes [8]. The ultra-intense $\left(\geq 10^{19} \mathrm{~W} / \mathrm{cm}^{2}\right)$ nature of the laser-target interaction foreseen to produce proton beams of energies suitable for hadrontherapy gives rise to ultra-short (from fs to ps) proton bursts at ultra-high dose rates $\left(\geq 10^{9} \mathrm{~Gy} / \mathrm{s}\right)$, i.e. several orders of magnitude larger than the ones used in the current therapeutic regimes [9]. While laser-driven particle beams can be thus seen as a valuable probe to investigate the core mechanisms of radiation 
action at the sub-nanometer and femtosecond scales [10,11], it has been suggested that the extreme spatio-temporal nature of laser-driven particle beams may ensue unknown biological responses $[7,12,13]$. Hence, ultra-fast high-energy particle radiobiology will have to ascertain whether and to what extent cellular radiosensitivity is influenced by such peculiar irradiation regimes. Whereas local tumour control is the aim of any curative radiotherapy, late-occurring effects such as recurrence or metastatization represent its most feared consequence. These stem from cells that are sub-lethally damaged by irradiation and by the interplay between these and neighbouring cancer cells. In particular, very little experimental data exist on the induction of sub-lethal cytogenetic damage along the Bragg curve, adding to the uncertainties deriving from still scant clinical follow-up data on sequelae incurred by hadrontherapy patients. A fortiori, the dependence of sub-lethal effects on unprecedented factors, such as the exceedingly high dose rates and/or the pulsed nature of beams originated by laser interaction with target materials, needs investigation prior to actual laser-driven cancer hadrontherapy. Here we present preliminary work carried out at LULI PICO $_{2000}$ laser facility focusing on cellular premature senescence in a normal human cell line.

\section{The radiobiology of ultra-fast high-energy particles}

Because of the increasing attention towards alternative modalities for cancer hadrontherapy, there has been a revival in recent years in the field of ultra-high dose rate (UHDR) radiobiology. In fact, an ample spectrum of dose rate values has been historically used in experimental radiobiology and clinical radiotherapy [14], extending from a few cGy per day to hundreds of Gy in a fraction of a second (Figure 1). The extremes of the spectrum have bene largely considered of mere academic interest as has been the case for the low end of the spectrum, i.e. chronic exposure regimes typical of interstitial brachytherapy, also of relevance to environmental exposure.

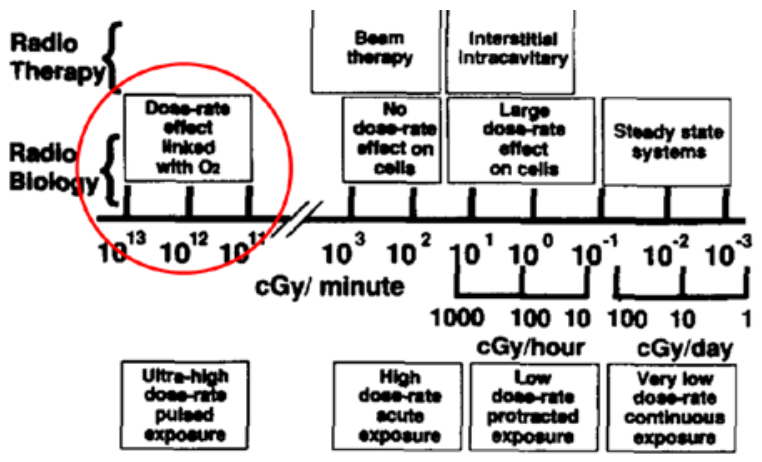

Figure 1: A sketch of the dose-rate spectrum of interest in radiobiology and radiotherapy: highlighted is the mechanistic basis hypothesized for biological effects due to UHDR and/or highly pulsed radiation regimes (adapted from [14])

Indeed, exploration of the top range of the dose.rate spectrum was long hampered by the technical difficulty of achieving such extreme conditions. The most complete set of data concerning the dose-rate effect has been accumulated in vitro for a number of biological systems in the range between $0.1 \mathrm{~Gy} / \mathrm{hr}$ and $10 \mathrm{~Gy} / \mathrm{min}$, demonstrating the principle of repair of sub-lethal damage $(15,16)$. Oxygen concentration was seen to be critical as an increase in cell survival with decreasing dose rates was seen to be much reduced under hypoxic conditions [17], 
pointing to less recoverable, intra-track lethal damage. At the opposite end of the dose-rate range, early studies invoked oxygen consumption by radiation concomitantly with an increased likelihood of recombination during free radical formation to explain a diminished biological effectiveness under UHDR [18]. UHDR were attained by discharge machines or electron linear accelerators: by both methods, estimated instantaneous dose rates of $10^{11} \mathrm{~Gy} / \mathrm{min}$ could be obtained [14]. If both the dose and instantaneous dose rate are sufficiently high, the rapid deposition of radiant energy depletes oxygen too quickly for diffusion to maintain an adequate level of oxygenation, and dose-response curves obtained are those characteristic of hypoxia, i.e. lacking the shoulder at low dose where cell killing is inefficient because of sub-lethal damage repair. The promise that these findings held for radiotherapy was based on the thought that UHDR would constitute a means of killing cells independent of oxygen tension since at sufficiently high dose rates even aerated cells would behave as if they were anoxic. This would represent an ideal radiation modality for radiotherapy since the protection afforded to some tumour cells by their hypoxia would be completely eliminated. For bacteria, a firmly established dose-rate effect in the UHDR region exists as first demonstrated by an anoxic-like response of bacteria irradiated in $1 \%$ oxygen [19]. This biphasic type of response was confirmed by others [20] irradiating mammalian cells equilibrated in air (Figure 2). Nias et al. showed instead lack of anoxic-like response and no differences in radiosensitivity after irradiation with highly pulsed electrons of oxyc cells [21]. Further studied failed to clarify the influence of UHDR and pulsed irradiation modality on cellular response. Schulz et al. [22] found a pulsed 30-MeV electron beam at $0.5-510^{8} \mathrm{~Gy} / \mathrm{s}$ to be less effective at cell killing compared to $250 \mathrm{kV}$ X-rays, but they could not relate their results to oxygen consumption. Purdie et al. [23] found opposite results using similar experimental conditions. These conflicting results, together with the consideration that total doses required to use up local oxygen are far too high, and the instantaneous dose-rates enormous, led to a decline of interest in this development for the clinic. At least, prior to the advent of laser-driven accelerating techniques.

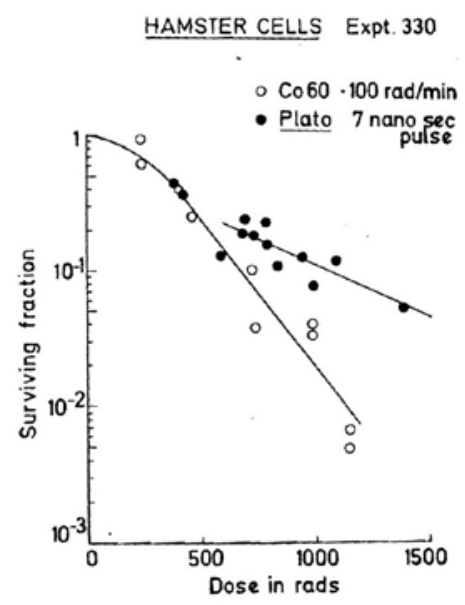

Figure 2: The typical "hockey-stick" type shape of the clonogenic survival dose response curves from $\gamma$-rays at conventional dose rate (1 Gy $\left.\mathrm{min}^{-1}\right)$ or to a 7-ns pulse of electrons (from [19]).

Several groups have started preliminary work using laser-driven ion sources for cell irradiation experiments. The main aim of these investigations so far has been to establish a procedure for cell handling, irradiation and dosimetry compatible with a laser-plasma interaction environment. Activities in this area have been carried out in Germany [24-26] and Japan [27], where, using 
Ti:Sa lasers, lethal effects on cells have been investigated by accumulating dose on cells over several laser shots, with an effective dose rate of Gy/s or Gy/min. An UHDR radiobiology program was initiated on the TARANIS and GEMINI laser systems in the UK, employing a single exposure approach (single-shot irradiations), which is believed to be more relevant to isolate effects associated to short burst deposition, as the dose required to induce biological effects is deposited in a single, ultrashort burst [28, 29]. Neither approach did find significant radiobiological differences between laser-driven electrons or protons and conventionally accelerated beams or reference photon radiations. In fact, the majority of in vitro studies making use of different sources confirm that in the therapeutically relevant dose range of a few Gy, even if applied in a single pulse of only few nanoseconds duration, non-linear radiobiological effects due to simultaneous multiple damages in cells and, thus, below any timescale of repair mechanisms, are unlikely to arise [30-35]. Only two relevant exceptions exist: Achayra et al. [36], who reported a decrease in genetic damage measured as micronucleus formation after a single pulse of electrons but not after multiple pulses $\left(10^{6}\right.$ to $\left.10^{8} \mathrm{~Gy} / \mathrm{s}\right)$, hypothesising more efficient radical recombination; Schmid et al. [37], who found a slight decrease in effectiveness at causing (some types of) chromosome aberrations after nanopulsed protons (conventionally accelerated). Of fundamental importance to gain insights into laser-driven particle biological effectiveness is, of course, in vivo work. From these studies, mainly limited to tumour growth delay as endpoint of clinical relevance and still too scarce for firm conclusions to be drawn, no enhancing effects have emerged for both protons and electrons [38-40].

\section{Sub-lethal cellular damage and particle therapy}

Despite allowing considerable normal tissue sparing, a main health concern of hadrontherapy remains the onset of secondary cancers [41]. The tumorigenic potential of ionizing radiation has been long known as well as its dependence on dose, dose rate and radiation quality [42]. However, non-cancer late effects may also undermine overall treatment success. Recent evidence suggests a significantly higher risk of developing cardiovascular disease in patients cured for breast cancer, in which heart and/or blood vessel are deemed to receive some dose, which is in line with the results from the epidemiological studies on the Japanese atomic bombs survivors [43]. Stress-induced premature senescence (SIPS), a process whereby sub-lethally damaged cells enter a permanent state of quiescence, has attracted growing interest for its possible long-term health consequences [44, 45]. This is opposed to the well-known phenomenon of in vitro replicative senescence, which is the physiological exhaustion of a cell's ability to proliferate [46], mechanistically linked with the progressive shortening of endchromosome sequences (telomeres) [47], and universally recognised as a natural barrier to tumorigenesis in vivo [48,49]. In particular, ionizing radiation is capable of inducing SIPS and, more interestingly, charged particles have proven particularly effective at eliciting a senescence response at very low dose and dose rates [50, 51]. Ectopic accumulation in vivo of senescing cells may adversely affect normal tissue/organ integrity and/or function resulting in genomic instability and increased risk of cancer [52-54]. In hadrontherapy patients, the role of SIPS is still undetermined $[55,56]$. In addition, because senescing cells remain metabolically active [46], a Senescence -Associated Secretory Phenotype (SAPS) has been identified whose effect on neighboring primary cancer cells is controversial as secretion of factors released by cells undergoing SIPS has been associated with either the inhibition or the promotion of proliferation/invasiveness [57-59]. Therefore, sub-lethal effects clearly play a role as important as tumor local control in determining the successful outcome of cancer radiation treatment. To 
date, there is no knowledge on the influence that very high dose rates or pulsed time-structure in dose delivery may have on such effects.

\section{Radiobiological studies with laser-accelerated protons at the PICO2000 facility}

\subsection{Experimental setup}

Protons were generated from the interaction of laser bursts focused on 25 and $50 \mu \mathrm{m}$ Au foil targets. Laser energy was, on average, of $(100 \pm 10) \mathrm{J}$ with a pulse duration of $1.3 \mathrm{ps}$ ( mean laser intensity of approximately $5 \times 10^{19} \mathrm{~W} / \mathrm{cm}^{2}$ ). A dipole deflected the plasma-accelerated protons as to angularly disperse them according to their energy. Parasitic X-ray radiation was suppressed by appropriate lead shielding in the chamber, while X-rays were spatially separated from the deflected protons. A purposely designed cell dish was used for biological sample irradiation, with cells growing on its $3-\mu$ m mylar basis. To optimize sample irradiation, we simultaneously exposed three cell dots in one shot (on the same dish), each at a specific energy and dose (Figure 3).
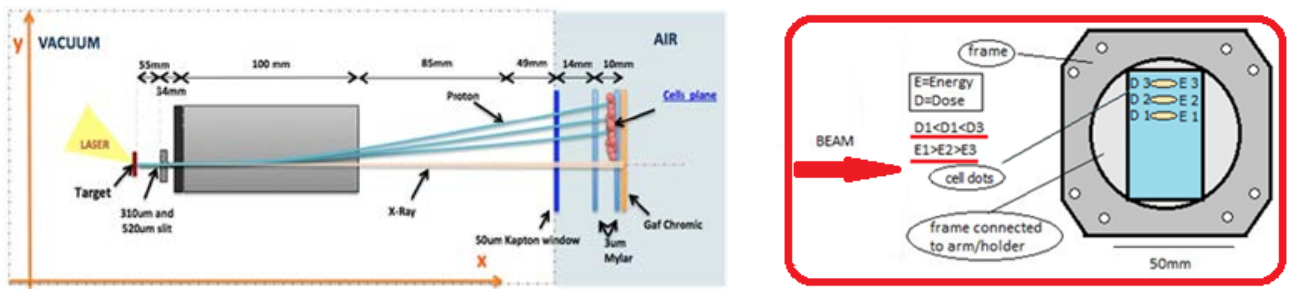

Figure 3: Proton beam transport and delivery system (left); Scheme of the cell dish and cell positioning(right)

After exposure, cells from each dot were treated independently. Radiochromic films (RCF), image plates (IP) and nuclear track detectors (CR39) were used for dosiemtry. Prior to cell irradiation, low-sensitivity HD-V2 RCF were positioned inside the target chamber at about $5 \mathrm{~cm}$ from the proton source, in order to study the beam input properties, in terms of energy and angular distribution. High-sensitivity EBT3 RCFs were placed outside the target chamber, just behind the biological sample, in an ad hoc frame, as to provide a shot-to-shot characterization of the dose. IP were employed to directly assess the energy distribution and its spatial uniformity at the sample position. CR39, instead, were used to estimate the particles fluence and homogeneity, and to cross-calibrate the IP. The energy differences within the dot-containing dish region varied in a range of 6 to $14 \mathrm{MeV}$, with a $10 \%$ dispersion approximately, corresponding to a biologically irrelevant LET variation ( $5 \pm 2 \mathrm{keV} / \mathrm{um}$ ). Hence, it was possible for the radiobiological data to be grouped by dose. Human Umbilical Vein Endothelial (HUVEC) cells were aliquoted as three dots of $20 \mu \mathrm{l}$ each of cell suspension, corresponding approximately to 15.000 cells, and seeded onto the cell dish $24 \mathrm{~h}$ before exposure. The three mean doses estimated at the cell positions were (0.6 \pm 0.2$) \mathrm{Gy},(1.8 \pm 0.6) \mathrm{Gy}$ and $(4.5 \pm 0.8) \mathrm{Gy}$. The onset of cellular senescence in irradiated cells was assessed by affinity for $\beta$-galactosidase expression, both at early time post-irradiation (2 days) and at later times. Clonogenic survival was also studied as it represents the standard measure for cell radiosensitivity. Results were compared against data from experiments with conventionally accelerated protons (mid-SOBP, LET $=5 \mathrm{keV} / \mu \mathrm{m}$ ) and X-rays carried out at INFN-LNS Catania and University of Naples, respectively. 


\subsection{Results}

Surviving fractions (SF) were fitted according to the linear-quadratic model SF=exp- $\left(\alpha D-\beta D^{2}\right)$. No statistically significant difference between cellular survival to laser-driven and conventionally accelerated proton beams was observed (Figure 4). An RBE of 1.1 was found between conventional proton irradiation and X-rays, in line with the universally accepted value in protontherapy planning treatments. Figure 5 illustrates the time course of radiation-induced cellular senescence. All irradiation conditions resulted in an increase in the fraction of senescing cells. In particular, proton irradiations were generally more effective than X-rays. As for laserdriven and conventionally accelerated protons, the former appeared less effective at the lowest dose, of relevance for healthy tissues in a radiotherapy scenario. Conversely, at the intermediate dose used in our experiments (1.5 Gy, 1.8 $\pm 0.5 \mathrm{~Gy}$ for laser-driven protons), i.e. a value close to the fractionated therapeutic dose of 2 Gy administered to the tumor volume, occurrence of senescence induced by laser-driven protons was at all times greater than that by conventionally accelerated protons . At the highest dose used (4 Gy, 4.5 $\pm 0.8 \mathrm{~Gy}$ for laser-driven protons) there appears to be no particular trend and little difference is noted between all radiation types.

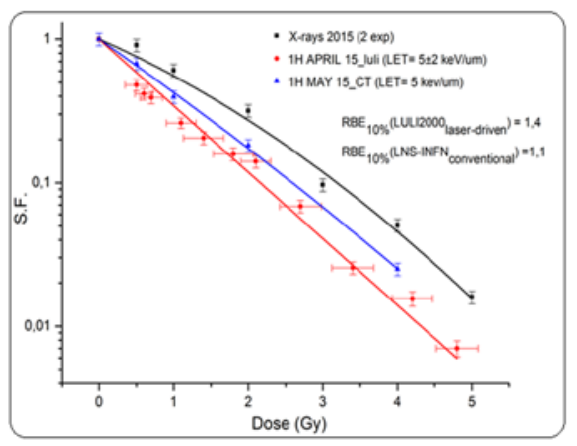

Figure 4: HUVEC clonogenic survival curves following laser-driven beam (red symbols) and conventionally accelerated beam (blue symbols). Results of X-ray irradiation (black symbols) are shown as a reference for RBE, which was estimated at the $10 \%$ survival level for all irradiation conditions.

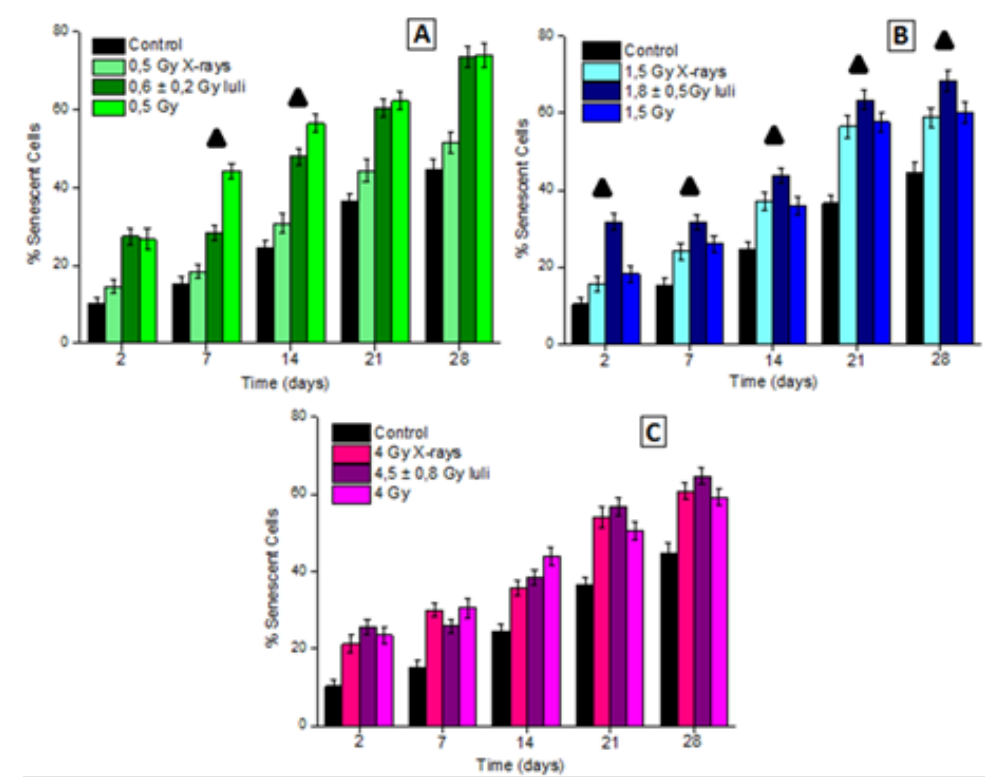

Figure 5: Time course of HUVEC senescence induction grouped by doses. Black triangles highlight the statistically significant differences between laser-driven and conventionally accelerated protons $(p<0.05)$. 


\subsection{Conclusions}

To our knowledge, the data here presented are the first ones obtained on radiation-induced premature senescence, an important sub-lethal effect of potential clinical relevance, in normal cells exposed to laser-accelerated particles. Laser-driven proton beams were found to yield a higher percentage of senescing cells at quasi-therapeutic doses while causing a lower percentage of cells to enter prematurely senescence at doses typical of healthy tissues. The potential clinical implications are intriguing since a greater effect at the tumor level (in vivo senescing endothelial cells may translate in disruption of the blood vessels feeding the tumor) seems to be accompanied by a lessened effect on normal tissues.

\section{References}

[1]. Particle Therapy Co-Operative Group (PTCOG). https://www.ptcog.ch/index.php/facilities-in operation. Last accessed January 112017.

[2]. W. D. Newhauser and R. Zhang, The physics of proton therapy, Phys. Med. Biol. 60 (2015) R155.

[3]. M. Pinto et al., Evidence for complexity at the nanometer scale of radiation-induced DNA DSBs as a determinant of rejoining kinetics, Radiat. Res. 164 (2005) 73.

[4]. A. Facoetti et al., In vivo radiobiological assessment of the new clinical carbon ion beams at CNAO, Radiat. Prot. Dosimetry 166 (2015) 379.

[5]. A. Peeters et al., How costly is particle therapy? Cost analysis of external beam radiotherapy with carbon-ions, protons and photons, Radiother. Oncol. 95 (2010) 45.

[6]. R. Bingham R, Basic concepts in plasma accelerators, Philos. Trans. A Math. Phys. Eng. Sci. 364 (2006) 559.

[7]. $\quad$ K.W.D. Ledingham et al., Towards Laser Driven Hadron Cancer Radiotherapy: A Review of Progress, Appl. Sci. 4 (2014), 402.

[8]. J. F.Ward, DNA damage produced by ionizing radiation in mammalian cells: identities, mechanisms of formation, and reparability, Prog. Nucleic Acid Res. Mol. Biol. 35 (1988) 95.

[9]. F. Schillaci et al. ELIMED, MEDical and multidisciplinary applications at ELI-Beamlines Journal of Physics: Conference Series, 508 (2014) 012010.

[10]. J. V. Malka, Ultra-short electron beams based spatio-temporal radiation biology and radiotherapy, Mutat Res. 704 (2010) 142.

[11]. B. Dromey et al., Picosecond metrology of laser-driven proton bursts, Nat Commun 7 (2016) 10642.

[12]. P. Wilson et al., Revisiting the ultra-high dose rate effect: implications for charged particle radiotherapy using protons and light ions, Br. J. Radiol. 85 (2012) e933.

[13]. C. Obcemea, Potential clinical impact of laser-accelerated beams in cancer ion therapy, NIM A 829 (2016) 149.

[14]. E. J. Hall and D. J. Brenner, The dose-rate effect revisited: radiobiological considerations of importance in radiotherapy, Int. J. Radiat. Oncol. Biol. Phys. 21 (1991) 1403.

[15]. E. J. Hall and J. S. Bedford, Dose-rate: its effect on the survival of HeLa cells irradiated with gamma-rays. Radiat. Res. 22 (1964) 305.

[16]. M. M. Elkind and H. Sutton, X-ray damage and recovery in mammalian cells in culture. Nature, 184 (1959) 1293.

[17]. E. J. Hall and J. Cavanagh, The oxygen effect for acute and protracted radiation exposures measured with seedlings of Vicia faba. Br. J. Radiol. 40 (1967) 128.

[18]. C. D. Town, Effect of high dose-rates on survival of diation with tritiated water ofmammalian cells in a mono-mammalian cells. Nature, 215 (1967) 847.

[19]. D. L. Dewey and J. W. Boag, Modification of the oxygen effect when bacteria are given large doses of radiation, Nature, 183 (1959) 1450. 
[20]. R. J. Berry et al., Survival of mammalian cells exposed to X rays at ultra high dose-rates. Br. J. Radiol. 42 (1969) 102.

[21]. A.H. Nias et al., Survival of HeLa cells from 10 nanosecond pulses of electrons, Int. J. Radiat. Biol. Relat. Stud. Phys. Chem. Med. 17 (1970) 595-598

[22]. $\quad$ R. J. Schulz et al., The effects of ultra-high dose rates on survival and sublethal repair in Chinese-hamster cells, Int. J. Radiat. Biol. Relat. Stud. Phys. Chem. Med. 33 (1978) 81.

[23]. W.J. Purdie et al., Increased Sensitivity of Mammalian Cells Irradiated at High Dose Rates under Oxic Conditions, Int. J. Radiat. Biol. 37 (1980) 331.

[24]. S. D. Kraft et al., Dose-dependent biological damage of tumour cells by laser-accelerated proton beams, New J. Phys. 12 (2010) 085003.

[25]. C. Richter et al., A dosimetric system for quantitative cell irradiation experiments with laseraccelerated protons, Phys. Med. Biol. 56 (2011) 1529.

[26]. K. Zeil et al., Dose-controlled irradiation of cancer cells with laser-accelerated proton pulses, Appl. Phys. B 110 (2013) 437.

[27]. A. Yogo et al., Measurement of relative biological effectiveness of protons in human cancer cells using a laser-driven quasimonoenergetic proton beamline, Appl Phys Lett. 98 (2011) 053701.

[28]. D. Doria et al., Biological effectiveness of live cells of laser driven protons at dose rates exceeding $10^{9} \mathrm{~Gy} / \mathrm{s}$, AIP Advances 2 (2012) 011209.

[29]. J. Bin et al., A laser-driven nanosecond proton source for radiobiological studies, Appl. Phys. Lett. 101 (2012) 243701.

[30]. T. E. Schmid et al., No Evidence for a Different RBE between Pulsed and Continuous $20 \mathrm{MeV}$ Protons, Radiat. Res. 172 (2009) 567.

[31]. T. E. Schmid et al., Relative biological effectiveness of pulsed and continuous $20 \mathrm{MeV}$ protons for micronucleus induction in 3D human reconstructed skin tissue, Radiother. Oncol. 95 (2010) 66.

[32]. S. Auer et al., Survival of tumor cells after proton irradiation with ultra-high dose rates, Radiat Oncol 6 (2011) 139.

[33]. L.Laschinsky et al., Radiobiological Effectiveness of Laser Accelerated Electrons in Comparison to Electron Beams from a Conventional Linear Accelerator, J. Radiat. Res. 53 (2012) 395.

[34]. O. Zlobinskaya et al., Induction and repair of DNA double-strand breaks assessed by gammaH2AX foci after irradiation with pulsed or continuous proton beams, Radiat. Environ. Biophys. 51 (2012) 23.

[35]. $\quad$ E. Beyreuther et al., Radiobiological response to ultra-short pulsed megavoltage electron beams of ultra-high pulse dose rate, Int. J. Radiat. Biol. 91 (2015) 643.

[36]. S. Acharya et al., Dose rate effect on micronuclei induction in human blood lymphocytes exposed to single pulse and multiple pulses of electrons, Radiat. Environ. Biophys 50 (2011) 253.

[37]. T. E. Schmid et al., The Effectiveness of $20 \mathrm{MeV}$ Protons at Nanosecond Pulse Lengths in Producing Chromosome Aberrations in Human-Hamster Hybrid Cells, Radiat. Res. 175 (2011) 719.

[38]. O. Zlobinskaya et al., The Effects of Ultra-High Dose Rate Proton Irradiation on Growth Delay in the Treatment of Human Tumor Xenografts in Nude Mice, Radiat. Res. 181 (2014) 177.

[39]. K. Brüchner et al., Establishment of a small animal tumour model for in vivo studies with low energy laser accelerated particles Radiat Oncol.9 (2014) 57.

[40]. M. Oppelt et al., Comparison study of in vivo dose response to laser-driven versus conventional electron beam. Radiat Environ Biophys. 54 (2015) 155.

[41]. E.J. Hall, Intensity-modulated radiation therapy, protons and the risk of second cancers. Int. J. Radiation Oncology Biol. Phys. 65 (2006) 1.

[42]. T.C.-H. Yang et al., Dose protraction studies with low- and high-LET radiations on neoplastic cell transformation in vitro. Adv. Space Res. 6 (1986) 137.

[43]. M. Boerma et al., Effects of ionizing radiation on the heart, Mutat. Res. 770 (2016) 319. 
[44]. M. S. Goligorsky et al., Stress-induced premature senescence of endothelial cells: a perilous state between recovery and point of no return, Curr Opin Hematol. 16 (2009) 215.

[45]. D. Lowe et al., Epigenetic clock analyses of cellular senescence and ageing, Oncotarget 7 (2016) 8524.

[46]. L. Hayflick and P.S. Moorhead, The serial cultivation of human diploid cell strains. Exp. Cell Res. 25 (1961) 585

[47]. R. Hastings et al. (2004), Telomere attrition and accumulation of senescent cells in cultured human endothelial cells. Cell Prolif. 37 (2004) 317.

[48]. J. Campisi, Cellular senescence as a tumour-suppressor mechanism. Trends Cell Biol. 11 (2011) 27.

[49]. A. Pribluda et al. A senescence-inflammatory switch from cancer-inhibitory to cancer-promoting mechanism.Cancer Cell. 24 (2013) 242.

[50]. M. Suzuki, et al., Reduction in life span of normal human fibroblasts exposed to very low-doserate charged particles. Radiat Res. 164 (2005) 505.

[51]. C. Fournier et al. Interrelation amongst differentiation, senescence and genetic instability in long-term cultures of fibroblasts exposed to different radiation qualities. Radiother. Oncol. 83 (2007) 277.

[52]. M. Sedic et al., Haploinsufficiency for BRCA1 leads to cell-type-specific genomic instability and premature senescence, Nat Commun. 6 (2015) 7505.

[53]. R. M. Day et al., Radiation-induced accelerated senescence: A fate worse than death? Cell Cycle 13 (2014) 2011.

[54]. E. Kim et al., Senescent fibroblasts in melanoma initiation and progression: an integrated theoretical, experimental, and clinical approach. Cancer Res. 73 (2013) 6874.

[55]. M. Suzuki and D.A. Boothman (2008) Stress-induced premature senescence (SIPS) -influence of SIPS on radiotherapy. J. Radiat. Res. 48 (2008) 48, 105.

[56]. G. Grossi et al., Late cellular effects of 12C ions. Il Nuovo Cimento C 31 (2008) 39.

[57]. K. K. Tsai et al., Low-dose radiation-induced senescent stromal fibroblasts render nearby breast cancer cells radioresistant. Radiat Res 172 (2009) 306.

[58]. A. R. Davalos et al., Senescent cells as a source of inflammatory factors for tumor progression. Cancer Metastasis Rev. 29 (2010) 273.

[59]. J. Zhao et al., Quantitative Analysis of Cellular Senescence in Culture and In Vivo, Curr. Protoc. Cytom.79 (2017) 9.51.25. 